\title{
Axial superlocalization with vortex beams
}

\author{
D Koutný ${ }^{1}$, Z Hradil $^{1}$, J Řeháček ${ }^{1}$ and L L Sánchez-Soto ${ }^{2,3}$ \\ Department of Optics, Palacký University, 17. listopadu 12, 77146 Olomouc, Czech Republic \\ Departamento de Óptica, Facultad de Física, Universidad Computense, 28040 Madrid, Spain \\ Max-Planck-Institut für die Physik des Lichts, Staudtstraße 2, 91258 Erlangen, Germany \\ E-mail: rehacek@optics.upol.cz \\ 9 March 2021
}

\begin{abstract}
Improving axial resolution is of paramount importance for three-dimensional optical imaging systems. Here, we investigate the ultimate precision in axial localization using vortex beams. For Laguerre-Gauss beams, this limit can be achieved with just an intensity scan. The same is not true for superpositions of Laguerre-Gauss beams, in particular for those with intensity profiles that rotate on defocusing. Microscopy methods based on rotating vortex beams may thus benefit from replacing traditional intensity sensors with advanced modesorting techniques.
\end{abstract}

Keywords: quantum metrology, quantum Fisher information, axial resolution, superresolution

\section{Introduction}

Discerning the nanometer-scale details of living cells, tissues, and materials is of utmost importance for many modern research efforts. A trail towards to this holy grail was opened with the appearance of a set of methods, dubbed with the generic term of superresolution microscopy [1, 2], capable of bypassing the diffraction limit [3, 4, 5]: a barrier that was traditionally thought to be impenetrable.

A number of these techniques can also reveal three-dimensional (3D) structural details: relevant examples include stimulated-emission-depletion microscopy [6], PSF engineering [8, 9, 10, 11, 12], photoactivated-localization microscopy [7], and multiplane detection [13, 14, [15], to cite only but a few. All of them rely on a very accurate localization of point sources; they differ in how point objects are excited and how the corresponding emitted photons are collected.

For 3D imaging, the emitter is fluorescently labeled and determining its axial position is an indispensable ingredient. This problem has been throughly examined and some impressive results have been demonstrated so far [16]. However, the fundamental depth precision achievable by any such engineering method has been considered only lately [17, 18, 19]. The rationale behind is to make a systematic use of the quantum Fisher information (QFI) [20] and the associated quantum Cramér-Rao bound (QCRB) to get a measurement-independent limit [21, 22]. This is much along the lines of the work of Tsang and coworkers to quantify the transverse two-point resolution [23, 24, 25, 26, 27], which has led to the dispelling of the Rayleigh curse [28, 29, 30, 31]. 
In a recent work [32], the ultimate precision in axial localization using Gaussian beams has been established. This limit can be attained with just one intensity scan, as long as the detection plane is placed at one optimal position.

In this paper, we generalize those results and derive quantum limits for axial localization with Laguerre-Gauss (LG) beams, which carry quantized orbital angular momentum [33]. Here, the beam waist acts as a realization of the light emitted by a point source after e.g. mode conversion. Another relevant situation is the reflection of the beam from a surface in surface topology measurements, etc. By linearly superposing different LG modes, one can realize beams with amplitude, phase, and intensity patterns that simply rotate, under free space propagation, maintaining the transverse shape. These rotating structures lie at the core of a variety of sensing techniques [34, 35, 36, 37].

We demonstrate that a meager part of the full (quantum) information is available in intensity scans and only a small fraction of this can be attributed to the rotation. This clearly confirms the potential of modal expansions inspired by quantum information protocols [38], which allow for reaching the QCRB and thus can be considered as the optimal measurement. Our results make $3 \mathrm{D}$ superresolution imaging more feasible and potentially useful for improving the resolution of optical microscopes.

\section{Theoretical model}

The problem we address here is to estimate the distance traveled by a vortex beam from the beam waist to an arbitrary detection plane. We thus consider the beam waist as an object whose axial distance is to be determined. In what follows, we shall represent the fields using Dirac notation, for it makes it straightforward to expand the theory to other types of light states.

We take the beam to be represented by the pure state $|\Psi(0)\rangle$, where $z=0$ denotes the position of the object plane. The axial displacement is thus characterized by a unitary operation

$$
|\Psi(z)\rangle=\mathrm{e}^{\mathrm{i} G z}|\Psi(0)\rangle,
$$

where the Hermitian operator $G$ is the generator. To pinpoint the specific form of $G$, it is appropriate to use the transverse-position representation $\Psi(x, y ; z)=\langle x, y \mid \Psi(z)\rangle$. It then follows directly from equation 2.1 that

$$
\mathrm{i} \partial_{z} \Psi(x, y ; z)=-G \Psi(x, y ; z) \text {. }
$$

On the other hand, vortex beams are solutions of the paraxial Helmholtz equation [39, 40]

$$
2 \mathrm{i} k \partial_{z} \Psi(x, y, z)=\nabla_{T}^{2} \Psi(x, y, z),
$$

where $k$ is the wavenumber and $\nabla_{T}^{2}=\partial_{x x}+\partial_{y y}$ is the transverse Laplacian. A direct comparison leads us to

$$
G \mapsto-\frac{1}{2 k} \nabla_{T}^{2} .
$$

The detection plane is placed at $z$, wherein we perform an arbitrary measurement. Given the formal analogies between spatial modes in wave optics and pure states in quantum theory, and also the mathematical similarities in describing evolution and detection of such objects, the amount of information about the axial distance $z$ carried by the measured signal is quantified by the QFI. For pure states, as it is our case, the QFI reduces to [21]

$$
\mathscr{Q}(z)=4 \operatorname{Var}(G) .
$$


Except for the factor 4, the QFI is the variance of the generator $G$ computed in the initial state $|\Psi(0)\rangle$. According to the time-honored QCRB, the variance of any unbiased estimator $\widehat{z}$ of the axial distance $z$ satisfies

$$
\operatorname{Var}(\widehat{z}) \geq \frac{1}{\mathscr{Q}(z)}
$$

whose saturation provides the ultimate precision in axial distance estimation.

To proceed further, we take the structure of the transverse field to correspond with LG modes

$$
\begin{aligned}
& \mathrm{LG}_{p l}(r, \phi, z)=\langle r, \phi, z \mid p, l\rangle=\sqrt{\frac{2 p !}{\pi(p+|l|) !}} \frac{1}{w(z)}\left[\frac{\sqrt{2} r}{w(z)}\right]^{|l|} \\
& \times L_{p}^{|l|}\left(\frac{2 r^{2}}{w(z)^{2}}\right) e^{-\frac{r^{2}}{w(z)^{2}}} \exp \left(\mathrm{i}\left[\frac{k r^{2}}{2 R(z)}-l \phi-\psi_{p l}(z)\right]\right),
\end{aligned}
$$

where $(r, \phi, z)$ are cylindrical coordinates, $L_{p}^{|l|}(\cdot)$ is the generalized Laguerre polynomial, $l \in\{0, \pm 1, \pm 2, \ldots\}$ is the azimuthal mode index and $p \in\{0,1,2, \ldots\}$ is the radial index. The parameters $R(z), w(z)$, and $\psi_{p l}(z)$ are

$$
\begin{aligned}
& R(z)=z\left[1+\left(z_{R} / z\right)^{2}\right] \\
& w^{2}(z)=w_{0}^{2}\left[1+\left(z / z_{R}\right)^{2}\right], \\
& \psi_{p l}(z)=(2 p+|l|+1) \arctan \left(z / z_{R}\right),
\end{aligned}
$$

and represent the radius of curvature of the wave front, the beam radius, and the Gouy phase [41], respectively, at an axial distance $z$ from the beam waist located at $z=0$. Here, $z_{\mathrm{R}}=k w_{0}^{2} / 2$ is the Rayleigh length and $w_{0}$ the beam waist radius [42].

\section{Quantum limit for axial localization with vortex beams}

To facilitate the derivation of the QFI corresponding to axial displacements of vortex beams it is advantageous to use an established correspondence between eigenstates of a twodimensional harmonic oscillator and paraxial beams [43]. For the case of LG beams, one defines

$$
a_{ \pm}=\frac{1}{\sqrt{2}}\left(a_{\xi} \mp i a_{\eta}\right)
$$

where $a_{\xi}$ and $a_{\eta}$ are dimensionless bosonic operators for each independent amplitude of the oscillator and obey the standard commutation relations $\left[a_{s}, a_{s^{\prime}}^{\dagger}\right]=\delta_{s s^{\prime}}\left(s, s^{\prime} \in\{\xi, \eta\}\right)$. Similar relations are obeyed by $a_{ \pm}$.

The eigenstates of the harmonic oscillator $\left|n_{+}, n_{-}\right\rangle$, generated by the action of $a_{+}^{\dagger}$ and $a_{-}^{\dagger}$ on the vacuum, are nothing but LG modes with azimuthal and radial indices given by

$$
l=n_{+}-n_{-}, \quad p=\min \left(n_{+}, n_{-}\right) .
$$

Applying the usual definition of the momentum operator $p_{s}=\frac{1}{\sqrt{2} \mathrm{i}}\left(a_{s}-a_{s}^{\dagger}\right)$, we have

$$
\widetilde{\nabla}_{T}^{2}=p_{\xi}^{2}+p_{\eta}^{2}=\left(p_{\xi}+i p_{\eta}\right)\left(p_{\xi}-i p_{\eta}\right)=\left(a_{+}-a_{-}^{\dagger}\right)\left(a_{+}^{\dagger}-a_{-}\right)
$$

If we recall (2.4), and take into account that, in the proper units, $(x, y) \mapsto$ $\left.\left(\sqrt{2} \xi / w_{0}, \sqrt{2} \eta / w_{0}\right\}\right)$, we get $\nabla_{T}^{2} \mapsto 2 \widetilde{\nabla}_{T}^{2} / w_{0}^{2}$. In this way, the QFI of a pure LG mode $\left|n_{+}, n_{-}\right\rangle$reads:

$$
\mathscr{Q}(z)=\frac{1}{z_{\mathrm{R}}^{2}}\left(2 n_{+} n_{-}+n_{+}+n_{-}+1\right)=\frac{1}{z_{\mathrm{R}}^{2}}[2 p(p+|l|)+2 p+|l|+1]
$$




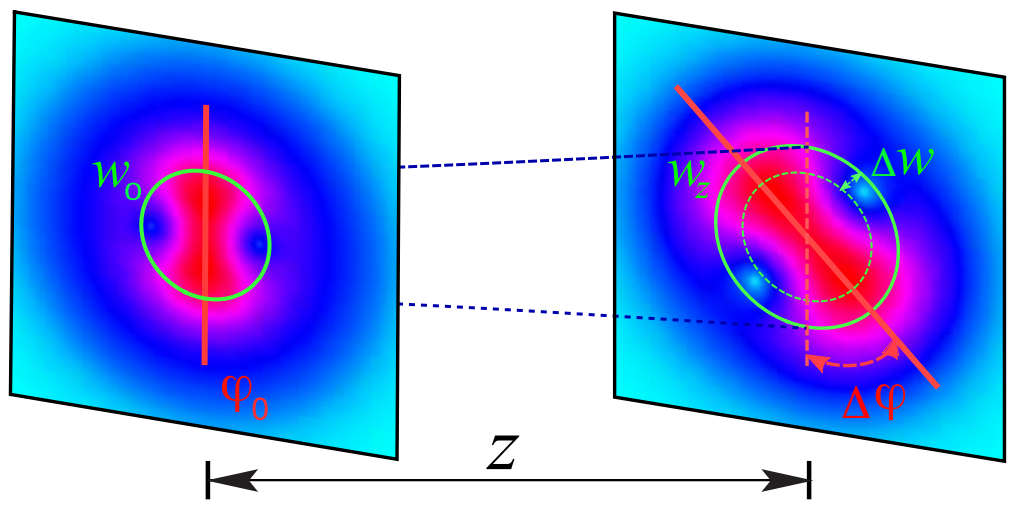

Figure 1. Sketch of the evolution of the superposition of two LG modes, with $l=2$ and $l^{\prime}=0$. The interference pattern is subject to rotation as well as divergence during propagation.

For the particular case of the Gaussian mode $\mathrm{LG}_{00}$ we get

$$
\mathscr{Q}(z)=\frac{1}{z_{\mathrm{R}}^{2}}
$$

that is, the quantum bound (per single detection) is precisely the Rayleigh range [44]. Note that the QFI is linear in $|l|$, which means that axial localization can be improved by using LG beams with large OAM.

Other sets of Gaussian transverse modes can be characterized using the so-called Hermite-Laguerre sphere [45, 46]. These modes are represented by a point, of spherical coordinates $(\theta, \phi)$, on that sphere, and they are generated by the rotated operators

$$
\begin{aligned}
& a_{1}(\theta, \phi)=a_{+} e^{-i \phi / 2} \cos \left(\frac{\theta}{2}\right)+a_{-} e^{i \phi / 2} \sin \left(\frac{\theta}{2}\right), \\
& a_{2}(\theta, \phi)=-a_{+} e^{-i \phi / 2} \sin \left(\frac{\theta}{2}\right)+a_{-} e^{i \phi / 2} \cos \left(\frac{\theta}{2}\right) .
\end{aligned}
$$

In particular, $\theta=\pi / 2$ gives rise to Hermite-Gauss (HG) modes, and $\theta=0, \pi$ to LG modes analyzed above. Combining equations (3.3) and (3.6), we get a direct generalization of (3.4); viz,

$\mathscr{Q}=\frac{1}{z_{\mathrm{R}}^{2}}\left[4+n_{1}+n_{2}\left(3+n_{2}\right)+n_{1}\left(3+4 n_{2}\right)+\left(n_{1}-n_{1}^{2}+n_{2}+4 n_{1} n_{2}-n_{2}^{2}\right) \cos (2 \theta)\right]$,

where $n_{1}$ and $n_{2}$ denote the eigenvalues of the corresponding number operators. Notice that the QFI (3.7) is independent of $\phi$ and is optimized by (3.4). This proves that LG modes are better than their HG counterparts, as axial localization is concerned.

Apart from a trivial divergence, transversal intensity profiles of pure vortex beams do not change on propagation. More complex intensity transformations can be realized by superposing two or more vortex beams. In particular, rotating structures are of interest in microscopy [34, 35, 36, 37]. Rotation of highly symmetric spots is easily detected and the corresponding defocusing parameter can be estimated from the measured rotation angle. This is roughly sketched in figure 1

To simplify the details as much as possible, we take the simple example of the superposition of two LG modes with different azimuthal numbers $l \neq l^{\prime}$, and $p=p^{\prime}=0$

$$
\left|\Psi_{l l^{\prime}}\right\rangle=\frac{1}{\sqrt{2}}\left(\left|\mathrm{LG}_{0 l}\right\rangle+\left|\mathrm{LG}_{0 l^{\prime}}\right\rangle\right)=\frac{1}{\sqrt{2}}\left(\left|n_{+}, 0\right\rangle+\left|n_{+}^{\prime}, 0\right\rangle\right) .
$$




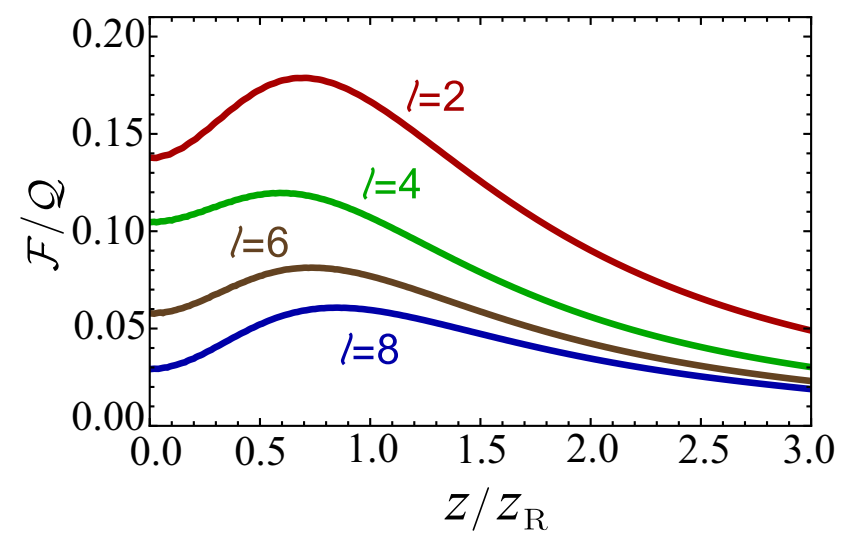

Figure 2. Fisher information about axial distance (relative to the QFI) for different superpositions $\left(\left|\mathrm{LG}_{0 l}\right\rangle+\left|\mathrm{LG}_{00}\right\rangle\right) / \sqrt{2}$ as a function of the position $z$ (relative to the beam waist) of the detection plane.

In this case, the QFI about the axial position of the source reads

$$
\mathscr{Q}=\frac{1}{z_{\mathrm{R}}^{2}}\left[4+2\left(|l|+\left|l^{\prime}\right|\right)+\left(|l|-\left|l^{\prime}\right|\right)^{2}\right] .
$$

Considering OAM as a resource for axial localization and using the maximum available OAM for one of the components of the superposition, $l= \pm\left|l_{\max }\right|$, the QFI is maximized when the second component is in the fundamental mode $l^{\prime}=0$, whereupon $\mathscr{Q}_{\max }=[4+$ $\left.2\left|l_{\max }\right|+\left|l_{\max }\right|^{2}\right] / z_{\mathrm{R}}^{2}$,and the quantum bound becomes quadratic in $|l|$. At first sight, the better performance of vortex superpositions (3.9) over pure vortex beams (3.4) is somehow related to beam rotation. This might suggest a proxy for the measured axial distance traveled from the waist to the detection plane. However, as we show in the next section, things are not that simple.

\section{Intensity detection}

In single-parameter estimation, the QFI can always be accessed and the corresponding QCRB saturated with a von Neumann measurement projecting the measured signal on the eigenstates of the symmetric logarithmic derivative of the density matrix [21]. In our context, the practical implementation of such measurements requires a spatial mode demultiplexer/sorter that performs simultaneous projection of the signal onto a complete orthonormal set of modes [47, 48]. Mode separation is usually achieved with a sequence of spatial light modulators implementing a suitable unitary transformation. Unavoidable systematic errors and losses introduced by such complicated experimental setups may ruin any theoretical advantage offered by optimal strategies.

In consequence, we consider the performance of the possibly inferior, but much more robust intensity detection, because it is the simplest method at hand for the experimentalist. As the information (3.4) and 3.7) about the axial distance is carried by both intensity and phase of the measured beam, we might aptly ask how much information is sacrificed by completely ignoring the phase. 
As usual, due to the noise, the detection can be considered as a random process. In consequence, the (normalized) beam intensity,

$$
p(r, \phi \mid z)=|\Psi(r, \phi ; z)|^{2},
$$

can be seen as the probability density of a detection conditional on the axial distance $z$. We take the detection as dominated by shot noise, which obeys a Poisson distribution [49]: although this neglects nonclassical effects, it is still a suitable model for realistic microscopy.

The classical Fisher information about $z$, per single detection, thus reads [50]

$$
\mathscr{F}=\int_{0}^{\infty} \int_{0}^{2 \pi} \frac{\left[\partial_{z} p(r, \phi \mid z)\right]^{2}}{p(r, \phi \mid z)} r d r d \phi
$$

and it is a suitable tool to quantify the information content about axial displacements accessible from the detected transversal intensity profile. For simplicity, we take the pixel size negligibly small, so that any sampling effect can be ignored. We also define the radial and azimuthal Fisher informations

$$
\begin{aligned}
& \mathscr{F}_{r}=\int_{0}^{\infty} \frac{\left[\partial_{z} \int_{0}^{2 \pi} p(r, \phi \mid z) d \phi\right]^{2}}{\int_{0}^{2 \pi} p(r, \phi \mid z) d \phi} r d r, \\
& \mathscr{F}_{\phi}=\int_{0}^{2 \pi} \frac{\left[\partial_{z} \int_{0}^{\infty} p(r, \phi \mid z) r d r\right]^{2}}{\int_{0}^{\infty} p(r, \phi \mid z) r d r} d \phi,
\end{aligned}
$$

respectively. They quantify the information of the radial and the azimuthal intensity components due to the $z$-dependence. For example, beam rotation only contributes to $\mathscr{F}_{\phi}$, whereas beam divergence contributes to $\mathscr{F}_{r}$.

For pure $\left|\mathrm{LG}_{p l}\right\rangle$ modes, $\Psi(r, \phi ; z)=\mathrm{LG}_{p l}(r, \phi, z)$, the classical Fisher information can be obtained in a closed form. First notice the integrand of (4.2) is independent of $\phi$. Carrying out $z$-derivatives of Laguerre polynomials using the relation $\partial_{z} L_{p}^{l}(z)=-L_{p-1}^{l+1}(z)$, and changing the integration variable $r$ yields

$$
\mathscr{F}=\frac{4 p !}{(|l|+p) !}\left[\frac{\partial_{z} w(z)}{w(z)}\right]^{2} \int_{0}^{\infty} e^{-t} t^{|l|}\left[2 t L_{p-1}^{|l|+1}(t)+(t-|l|-1) L_{p}^{|l|}(t)\right]^{2} d t
$$

We are thus left with evaluating six different integrals involving products of Laguerre polynomials. Compact expressions for each case can be obtained from the generalized orthogonality relation [51]

$$
\int_{0}^{\infty} \mathrm{e}^{-t} t^{\mu} L_{p}^{l}(t) L_{p^{\prime}}^{l^{\prime}}(t) \mathrm{d} t=(-1)^{p+p^{\prime}} \Gamma(\mu+1) \sum_{k=0}^{\min \left(p, p^{\prime}\right)}\left(\begin{array}{c}
\mu-l \\
p-k
\end{array}\right)\left(\begin{array}{c}
\mu-l^{\prime} \\
p^{\prime}-k
\end{array}\right)\left(\begin{array}{c}
\mu+k \\
k
\end{array}\right) .
$$

Straightforward simplifications leave us with the final result

$$
\mathscr{F}=4[2 p(p+|l|)+2 p+|l|+1]\left[\frac{\partial_{z} w(z)}{w(z)}\right]^{2}=\frac{2 p(p+|l|)+2 p+|l|+1}{R(z)^{2} / 4} .
$$

For the planes of maximal wavefront curvature $z_{\mathrm{opt}}= \pm z_{\mathrm{R}}$, where $R\left(z_{\mathrm{R}}\right)^{2}=4 z_{\mathrm{R}}^{2}$, the quantum limit is saturated with intensity sensitive detection

$$
\mathscr{F}_{\text {opt }}=\mathscr{Q} \text {. }
$$

Thus, for any pure LG beam and any beam waist location, two detection planes can be found, where complete information about the axial distance can be extracted with intensityonly detection. Hence, full potential of high-order vortex beams for axial metrology can be 


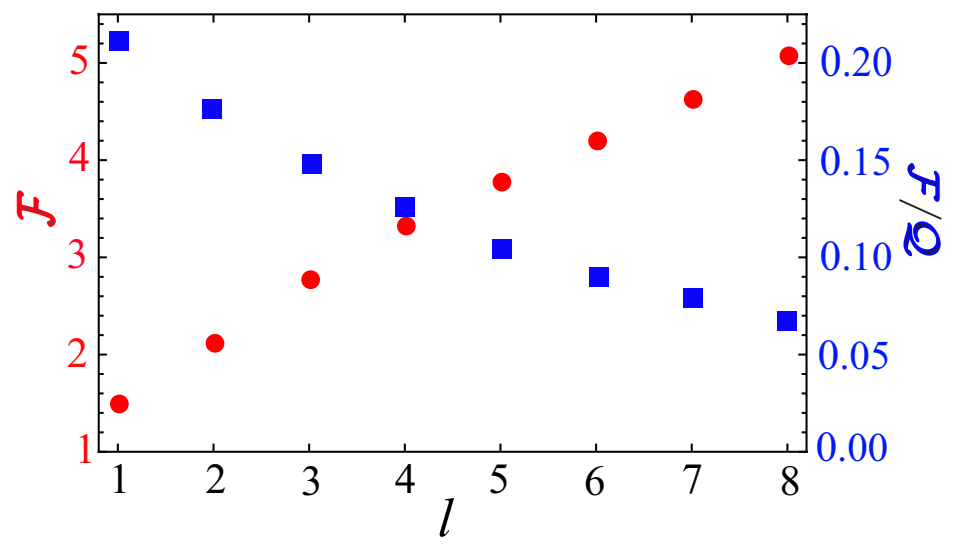

Figure 3. Red dots represent Fisher information about axial distance $z$ for optimally placed intensity detections for the superpositions $\left(\left|\mathrm{LG}_{0 l}\right\rangle+\left|\mathrm{LG}_{00}\right\rangle\right) / \sqrt{2}$. Blue squares are the same Fisher information, but normalized to the optimal QFI.

exploited with direct detection techniques. This generalizes the results obtained for Gaussian beams[32].

However, numerical analysis suggests that this result does not hold for superpositions of LG modes: a single transversal intensity scan is no longer optimal for any detector position z. In figure 2 we plot the classical Fisher information (normalized to the optimal QFI) for different vortex superpositions. First, notice that the optimal detector position is no longer at $z= \pm z_{\mathrm{R}}$, as it was for pure LG beams. Second, the larger the angular momentum $l$ carried by the signal, the smaller portion of the total QFI can be extracted with intensity measurements.

This is further illustrated in figure 3 . Locating intensity detectors at the optimal detection

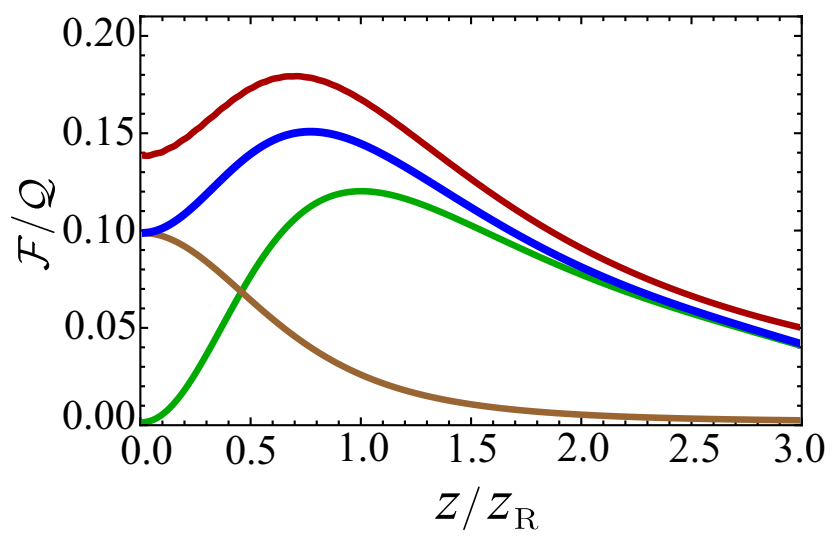

Figure 4. Radial (green), azimuthal (brown) and total (red) Fisher information $\mathscr{F}$ about axial displacement as a function of detector axial position $z$ (in units of $z_{\mathrm{R}}$ ) for the vortex superposition $\left(\left|\mathrm{LG}_{02}\right\rangle+\mathrm{LG}_{00}\right) / \sqrt{2}$. The sum of radial and azimuthal components is also shown (blue). All quantities are normalized to the corresponding optimal QFI. Notice that for small (large) propagation distances, most FI comes from azimuthal (radial) intensity distribution. Some information is stored in correlations between those two marginal distributions, as can be seen from the gap between the blue and the red curves. 
planes for every value of $l$, the classical Fisher information grows sublinearly with $l$, so that progressively smaller portion of quadratic QFI is available from intensity data and the gap between the quantum bound and the performance of the best estimator from intensity data worsens with $l$.

For example, for $l=2$, only about $17 \%$ of the QFI is available from intensity scan at the optimal detector position, as we can see in figure 4 Of this, only a small fraction is due to changes in the azimuthal intensity profile, as arises, e.g., in rotations, except for very small propagation distances, where azimuthal profile accounts for up to $10 \%$ of QFI. This ratio quickly approaches zero with increasing $l$.

The information content of rotating beam intensity distributions is surprisingly low. As bad as it sounds, this is not a negative result, but encouraging news for quantum metrology. It highlights inadequacy of simple detection techniques in this particular metrological scenario, where intensity detection fails to reveal all potentially accessible information about the parameter of interest. The hidden Information has to be accessed with advanced detection techniques. Here a generic tool is projecting/sorting the signal into optimal set of spatial modes derivable from the quantum detection theory. Hence, we uncover a huge potential of axial superlocalization based on spatial-mode projections applied to higher order vortex beam superpositions [44].

\section{Concluding remarks}

We have established the ultimate quantum limits for axial localization using vortex beams. For pure LG beams, this limit is attained with an intensity scan with the detector located at one of two optimal planes. For superpositions of LG beams, in particular of those with intensity profiles rotating on defocusing, this is no longer true. This means that microscopy methods based on rotating vortex beams may benefit from replacing traditional intensity scans with advanced mode-sorting techniques.

We thank Robert W. Boyd and Aaron Z. Goldberg for helpful discussions. We acknowledge financial support of the project ApresSF, under the QuantERA programme, which has received funding from the European Union's Horizon 2020 research and innovation programme, the program H2020 (project StormyTune), Grant Agency of the Czech Republic (Grant No. 18-04291S), the Palacký University (GrantNo. IGA_PrF_2020_004), and the Spanish MINECO (Grant PGC2018-099183-B-I00). 
[1] Focus issue: Super-resolution imaging. Nat. Photonics, 3:361-420, 2009.

[2] L. Schermelleh, A. Ferrand, T. Huser, C. Eggeling, M. Sauer, O. Biehlmaier, and G. P. C. Drummen. Superresolution microscopy demystified. Nat. Cell Bio., 21:72-84, 2019.

[3] E. Abbe. Ueber einen neuen Beleuchtungsapparat am Mikroskop. Arch. Mikrosk. Anat., 9:469-480, 1873.

[4] Lord Rayleigh. Investigations in Optics, with special reference to the spectroscope. Phil. Mag., 8:261-274, 403-411, 477-486, 1879.

[5] S. Ram, E. S. Ward, and R. J. Ober. Beyond Rayleigh's criterion: A resolution measure with application to single-molecule microscopy. PNAS, 103:4457-4462, 2006.

[6] S. W. Hell and J. Wichmann. Breaking the diffraction resolution limit by stimulated emission: stimulatedemission-depletion fluorescence microscopy. Opt. Lett., 19:780-782, 1994.

[7] E. Betzig, G. H. Patterson, R. Sougrat, O. W. Lindwasser, S. Olenych, J. S. Bonifacino, M. W. Davidson, J. Lippincott-Schwartz, and H. F. Hess. Imaging intracellular fluorescent proteins at nanometer resolution. Science, 313:1642, 2006.

[8] B. Huang, W. Wang, M. Bates, and X. Zhuang. Three-dimensional super-resolution imaging by stochastic optical reconstruction microscopy. Science, 319:810, 2008.

[9] S. R. P. Pavani, M. A. Thompson, J. S. Biteen, S. J. Lord, N. Liu, R. J. Twieg, R. Piestun, and W. E. Moerner. Three-dimensional, single-molecule fluorescence imaging beyond the diffraction limit by using a doublehelix point spread function. Proc. Natl. Acad. Sci. USA, 106:2995, 2009.

[10] S. Jia, J. C. Vaughan, and X. Zhuang. Isotropic three-dimensional super-resolution imaging with a self-bending point spread function. Nat. Photonics, 8:302, 2014.

[11] F. Tamburini, G. Anzolin, G. Umbriaco, A. Bianchini, and C. Barbieri. Overcoming the Rayleigh criterion limit with optical vortices. Phys. Rev. Lett., 97:163903, 2006.

[12] M. Paúr, B. Stoklasa, J. Grover, A. Krzic, L. L. Sánchez-Soto, Z. Hradil, and J. Řeháček. Tempering Rayleigh's curse with PSF shaping. Optica, 5:1177-1180, 2018.

[13] M. F. Juette, T. J. Gould, M. D. Lessard, M. J. Mlodzianoski, B. S. Nagpure, B. T. Bennett, S. T. Hess, and J. Bewersdorf. Three-dimensional sub-100 nm resolution fluorescence microscopy of thick samples. Nat. Methods, 5:527, 2008.

[14] P. A. Dalgarno, H. I. C. Dalgarno, A. Putoud, R. Lambert, L. Paterson, D. C. Logan, D. P. Towers, R. J. Warburton, and A. H. Greenaway. Multiplane imaging and three dimensional nanoscale particle tracking in biological microscopy. Opt. Express, 18:877-884, 2010.

[15] S. Abrahamsson, J. Chen, B. Hajj, S. Stallinga, A. Y. Katsov, J. Wisniewski, G. Mizuguchi, P. Soule, F. Mueller, C. D. Darzacq, X. Darzacq, C. Wu, C. I. Bargmann, D. A. Agard, M. Dahan, and M. G. L. Gustafsson. Fast multicolor 3D imaging using aberration-corrected multifocus microscopy. Nat. Methods, 10:60, 2012.

[16] A. von Diezmann, Y. Shechtman, and W. E. Moerner. Three-dimensional localization of single molecules for super-resolution imaging and single-particle tracking. Chem. Rev., 117:7244-7275, 2017.

[17] M. Tsang. Quantum limits to optical point-source localization. Optica, 2:646-653, 2015.

[18] R. Nair and M. Tsang. Interferometric superlocalization of two incoherent optical point sources. Opt. Express, 24:3684-3701, 2016.

[19] M. P. Backlund, Y. Shechtman, and R. L. Walsworth. Fundamental precision bounds for three-dimensional optical localization microscopy with Poisson statistics. Phys. Rev. Lett., 121:023904, 2018.

[20] D. Petz and C. Ghinea. Introduction to Quantum Fisher Information, volume 27, pages 261-281. World Scientific, 2011

[21] C. W. Helstrom. Quantum Detection and Estimation Theory. Academic, New York, 1976.

[22] A. S. Holevo. Probabilistic and Statistical Aspects of Quantum Theory. North Holland, Amsterdam, 2nd edition, 2003.

[23] M. Tsang, R. Nair, and X.-M. Lu. Quantum theory of superresolution for two incoherent optical point sources. Phys. Rev. X, 6:031033, 2016.

[24] C. Lupo and S. Pirandola. Ultimate precision bound of quantum and subwavelength imaging. Phys. Rev. Lett., 117:190802, 2016

[25] R. Nair and M. Tsang. Far-field superresolution of thermal electromagnetic sources at the quantum limit. Phys. Rev. Lett., 117:190801, 2016.

[26] S. Z. Ang, R. Nair, and M. Tsang. Quantum limit for two-dimensional resolution of two incoherent optical point sources. Phys. Rev. A, 95:063847, 2016.

[27] M. Tsang. Subdiffraction incoherent optical imaging via spatial-mode demultiplexing. New J. Phys., 19:023054, 2017.

[28] M. Paur, B. Stoklasa, Z. Hradil, L. L. Sanchez-Soto, and J. Rehacek. Achieving the ultimate optical resolution. Optica, 3:1144-1147, 2016

[29] F. Yang, A. Taschilina, E. S. Moiseev, C. Simon, and A. I. Lvovsky. Far-field linear optical superresolution via heterodyne detection in a higher-order local oscillator mode. Optica, 3:1148-1152, 2016.

[30] W. K. Tham, H. Ferretti, and A. M. Steinberg. Beating Rayleigh's curse by imaging using phase information. Phys. Rev. Lett., 118:070801, 2016. 
[31] F. Yang, R. Nair, M. Tsang, C. Simon, and A. I. Lvovsky. Fisher information for far-field linear optical superresolution via homodyne or heterodyne detection in a higher-order local oscillator mode. Phys. Rev. A, 96:063829, 2017.

[32] J. Řeháček, M. Paúr, B. Stoklasa, D. Koutný, Z. Hradil, and L. L. Sánchez-Soto. Intensity-based axial localization at the quantum limit. Phys. Rev. Lett., 123:193601, 2019.

[33] L. Allen, M. W. Beijersbergen, R. J. C. Spreeuw, and J. P. Woerdman. Orbital angular momentum of light and the transformation of Laguerre-Gaussian laser modes. Phys. Rev. A, 45:8185-8189, 1992.

[34] R. Piestun, Y. Y. Schechner, and J. Shamir. Propagation-invariant wave fields with finite energy. J. Opt. Soc. Am. A, 17:294-303, 2000.

[35] A. Greengard, Y. Y. Schechner, and R. Piestun. Depth from diffracted rotation. Opt. Lett., 31:181-183, 2006.

[36] S. Prasad. Rotating point spread function via pupil-phase engineering. Opt. Lett., 38:585-587, 2013.

[37] M. Baránek, P. Bouchal, M. Šiler, and Z. Bouchal. Aberration resistant axial localization using a self-imaging of vortices. Opt. Express, 23:15316-15331, 2015.

[38] C. Fabre and N. Treps. Modes and states in quantum optics. Rev. Mod. Phys., 92:035005, 2020.

[39] S. Franke-Arnold, L. Allen, and M. Padgett. Advances in optical angular momentum. Laser Photon. Rev., 2:299-313, 2008.

[40] S. M. Barnett, M. Babiker, and M. J. Padgett. Optical orbital angular momentum. Phil. Trans. Roy. Soc. A, 375:20150444, 2017.

[41] S. Feng and H. G. Winful. Physical origin of the Gouy phase shift. Opt. Lett., 26:485-487, 2001.

[42] A.E. Siegman. Lasers. Oxford University Press, Oxford, 1986.

[43] G. Nienhuis. Analogies between optical and quantum mechanical angular momentum. Phil. Trans. Roy. Soc. A, 375:20150443, 2017.

[44] Y. Zhou, J. Yang, J. D. Hassett, S. M. H. Rafsanjani, M. Mirhosseini, A. N. Vamivakas, A. N. Jordan, Z. Shi, and R. W. Boyd. Quantum-limited estimation of the axial separation of two incoherent point sources. Optica, 6:534-541, 2019.

[45] R. Simon and G. S. Agarwal. Wigner representation of Laguerre-Gaussian beams. Opt. Lett., 25:1313-1315, 2000.

[46] J. Visser and G. Nienhuis. Orbital angular momentum of general astigmatic modes. Phys. Rev. A, 70:013809, 2004.

[47] J. Rehacek, M. Paúr, B. Stoklasa, Z. Hradil, and L. L. Sánchez-Soto. Optimal measurements for resolution beyond the Rayleigh limit. Opt. Lett., 42:231-234, 2017.

[48] J. Rehacek, Z. Hradil, B. Stoklasa, M. Paúr, J. Grover, A. Krzic, and L. L. Sánchez-Soto. Multiparameter quantum metrology of incoherent point sources: towards realistic superresolution. Phys. Rev. A, 96:062107, 2017.

[49] D. R. Fuhrmann, C. Preza, J. A. O'Sullivan, D. L. Snyder, and W. H. Smith. Spectrum estimation from quantum-limited interferograms. IEEE Trans. Signal Process., 52:950-961, 2004.

[50] R. A. Fisher. Theory of statistical estimation. Math. Proc. Cambridge, 22:700-725, 1925.

[51] Th. M. Rassias and H. M. Srivastava, The orthogonality property of the classical Laguerre polynomials Appl. Math. Comput., 50:167-173, 1992. 